\title{
Dicer and Drosha expression and response to Bevacizumab-based therapy in advanced colorectal cancer patients
}

\author{
Bruno Vincenzi ${ }^{\mathrm{a}, *}$, Alice Zoccoli ${ }^{\mathrm{a}}$, Gaia Schiavon ${ }^{\mathrm{b}}$, Michele Iuliani ${ }^{\mathrm{a}}$, \\ Francesco Pantano ${ }^{a}$, Emanuela Dell'Aquila ${ }^{a}$, Raffaele Ratta ${ }^{a}$, Andrea Onetti Muda ${ }^{c}$, \\ Giuseppe Perrone $^{\mathrm{c}}$, Chiara Brunelli ${ }^{\mathrm{c}}$, Pierpaolo Correale ${ }^{\mathrm{d}}$, Elisabetta Riva ${ }^{\mathrm{e}}$, \\ Antonio Russo ${ }^{\mathrm{f}}$, Fotios Loupakis ${ }^{\mathrm{g}}$, Alfredo Falcone ${ }^{\mathrm{g}}$, Daniele Santini ${ }^{\mathrm{a}}$, \\ Giuseppe Tonini ${ }^{\mathrm{a}}$
}

${ }^{a}$ Medical Oncology, University Hospital Campus Bio-Medico, Via Alvaro del Portillo 200, 00128 Rome, Italy

${ }^{\mathrm{b}}$ Department of Medical Oncology, Erasmus University Medical Center, Daniel den Hoed Cancer Center, 301 Groene Hilledijk, 3075 EA, Rotterdam, The Netherlands

${ }^{\mathrm{c}}$ Pathology Division, University Hospital Campus Bio-Medico, Via Alvaro del Portillo 200, 00128 Rome, Italy

d Oncopharmacology Center, School of Medicine, University of Siena, Viale Bracci 11, 53100 Siena, Italy

${ }^{\mathrm{e}}$ Virology Section, University Hospital Campus Bio-Medico, Via Alvaro del Portillo 200, 00128 Rome, Italy

${ }^{\mathrm{f}}$ Section of Medical Oncology, Department of Surgical and Oncological Sciences, University of Palermo, Via del Vespro 129, 90127 Palermo, Italy

${ }^{\mathrm{g}}$ Section of Medical Oncology 2, University Hospital of Pisa, Via Paradisa 2, 56100 Pisa, Italy

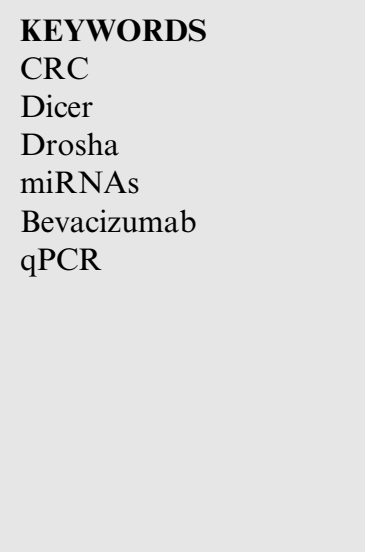

\begin{abstract}
Purpose: The miRNA-regulating enzymes Dicer and Drosha exhibit aberrant expression in several cancer types. Dicer and Drosha play a crucial role during the angiogenetic process in vitro and, for Dicer, in vivo. We aimed to investigate the potential role of Dicer and Drosha in predicting response to Bevacizumab-based therapy in advanced colorectal cancer (CRC) patients.

Methods: Dicer and Drosha mRNA levels were analysed in formalin-fixed paraffin-embedded specimens from patients affected by advanced CRC treated with or without Bevacizumabcontaining regimens ( $n=116$ and $n=50$, respectively) and from patients with diverticulosis as control group $(n=20)$. The experimental data were obtained using qRT-PCR, analysed comparing Dicer and Drosha expression levels in tumour samples versus normal mucosa and then compared to clinical outcome.

Results: The tumour samples from Bevacizumab-treated patients showed a significantly higher Drosha expression $(P<.001)$ versus normal mucosa, while Dicer levels did not differ. Intriguingly, we found that low Dicer levels predicted a longer progression-free survival (PFS)
\end{abstract}

* Corresponding author: Address: Campus Bio-Medico University of Rome, Department of Medical Oncology, Via Alvaro del Portillo 200, 00128 Rome, Italy. Tel.: +39 3346966435.

E-mail address: b.vincenzi@unicampus.it (B. Vincenzi). 
$(P<.0001)$ and overall survival $(\mathrm{OS})(P=.009)$. In addition, low Dicer levels were associated with better response to Bevacizumab-based treatments versus high Dicer levels $(1.7 \%$ complete responses and $53.4 \%$ partial responses versus $0 \%$ and $32.7 \%$, respectively; $P=.0067)$. Multivariate analysis identified three independent predictors of improved OS: high performance status (PS) (relative risk (RR) $1.45 ; P=.011$ ), lower organs involvement (RR $0.79 ; P=.034$ ) and low Dicer expression (RR $0.71 ; P=.008$ ). Conversely, Drosha levels were not associated with prognosis and outcome associated with treatment. In non-Bevacizumab-treated patients, Dicer and Drosha expression did not correlate with outcome.

Conclusion: These findings suggest that low Dicer mRNA levels seem to be independent predictors of favourable outcome and response in patients affected by advanced CRCs treated with Bevacizumab-based therapy.

(C) 2012 Elsevier Ltd. All rights reserved.

\section{Introduction}

Colorectal cancer (CRC) is the third world's leading cause of cancer death. About $40-50 \%$ of newly diagnosed patients account for a metastatic disease, which is associated with high mortality. ${ }^{1}$ The pathogenesis of CRC is a complex process, tightly controlled by multiple regulatory mechanisms including genome structure rearrangements, chromatin remodelling, epigenetic alterations and genetic mutations. ${ }^{2}$ In the past few years, a gradually increasing number of studies documented that these processes are regulated by a class of small noncoding RNAs called microRNAs (miRNA) and involved in a wide spectrum of biological processes. ${ }^{3}$

Recent evidences have shown that alteration in miRNA expression is involved in the pathogenesis of cancers and in the metastatization process. The master regulators of miRNA biogenesis are two ribonucleases called Dicer and Drosha that act at different stages of miRNA synthesis and maturation. In the "miRNA machinery", Drosha is involved in the initial step of miRNA processing in the nucleus, where short (60-70 nucleotides) double-stranded RNA precursors (pre-miRNAs) are generated. ${ }^{4}$

Subsequently, the resulting pre-miRNA is exported to the cytoplasm and then cleaved by Dicer to generate the mature products, double-stranded miRNA fragments of $15-30$ nucleotides. ${ }^{5-7}$

Some studies suggest that these factors, required for the biogenesis of miRNAs, are also implicated in cancer development. Growing evidences indeed show that Dicer and Drosha expression levels may vary among tumour types, but the regulation of these genes is still unclear. Recently, Karube et al. indicated that levels of Dicer could be used as prognostic markers in non-small cell lung cancer (NSCLC) and in breast cancer patients, showing that reduced messenger RNA (mRNA) expression is significantly associated with poor patient survival. $^{8,9}$ Moreover, Merritt et al. demonstrated that levels of Dicer and Drosha are prognostic factors in patients with ovarian cancer. ${ }^{10}$

In CRC, it has been demonstrated that a high expression (both at mRNA and protein level) of Dicer is signif- icantly related to poor survival, independent of gender, age, tumour site, stage and differentiation. ${ }^{11,12}$

Intriguingly, several studies have shown that Dicer and Drosha play a crucial role during the angiogenic process in vitro and that Dicer is also involved in the angiogenesis regulation in in vivo models. ${ }^{13}$ In fact, genetic silencing of Dicer in a mouse model was found to impair normal morphogenesis and organ development due to a de-regulation of angiogenesis-related genes. ${ }^{14}$

Nowadays Bevacizumab, a humanised recombinant monoclonal antibody that inhibits vascular endothelial growth factor A (VEGF-A), is part of the standard first-line treatment for metastatic CRC. ${ }^{15,16}$

Based on these data, our aim was to investigate the expression of Dicer and Drosha and their role as prognostic and predictive factors of response to $\mathrm{Bev}$ acizumab-based treatment in advanced CRC patients.

\section{Patients and methods}

\subsection{Exploratory review of microarray data}

We decided to query the cancer microarray database Oncomine $^{\mathrm{TM}}$ (Compendia Bioscience, Ann Arbor, MI, USA, version 4.4) for the mRNA expression of Dicer and Drosha, in order to have a large overview of the expression of our genes of interest across existing datasets. We decided to set a threshold $P$-value of 0.05 and fold change of 2 in order to include comparisons in our exploratory analysis. A gene/probe had to appear in the top $10 \%$ of the ranking to include the series in the analysis. The co-expression analysis in the significant series was also considered.

\subsection{Study population}

In our study we retrospectively included three different groups of patients, seen at the Campus Bio-Medico University of Rome (Departments of Medical Oncology and General Surgery) and affected by: (1) advanced CRC treated with Bevacizumab-containing regimens, (2) advanced CRC treated with not Bevacizumab-con- 
taining regimens and (3) diverticulosis treated with colorectal surgery (control group). For all patients, formalin-fixed paraffin-embedded (FFPE) surgical specimens, collected prior to start of any therapy and clinico-pathological data were available. Clinical response to therapy was based on RECIST criteria. Exclusion criteria were preoperative (neo-adjuvant) chemotherapy and/or radiotherapy.

Moreover, we collected peritumoural samples from a subgroup of patients who received Bevacizumab-containing regimens.

Samples were collected from January 2009 to December 2010; the median follow-up of patients was 21 months. Primary endpoints were to evaluate a potential association between the modulation of Dicer and Drosha expression levels and progression free survival (PFS), response rate and overall survival (OS). The other prognostic variables tested were: tumour grading, liver involvement, number of involved organs, performance status (PS), albumin, alkaline phosphatase, gamma-GT, LDH and basal CEA levels. REMARK criteria were satisfied.

\subsection{RNA extraction and gene expression analysis}

FFPE sections were treated with xylene and ethanol to remove paraffin; the tissue was dried and resuspended in Digestion Buffer and Proteinase K (Qiagen, UK) to allow sample lysis. The tissue was digested overnight at $56{ }^{\circ} \mathrm{C}$ and total RNA was extracted using the TRizol reagent (Invitrogen, CA, USA) according to the manufacturer's instructions. RNA was treated with DNase Buffer and DNase (DNAse Turbo, Applied Biosystems, CA, USA) to avoid genomic DNA contamination. The concentration and purity of the isolated RNA (A260/ A280 ratio between 1.8 and 2.0 were accepted) were measured using a NanoDrop ND-1000 Spectrophotometer (Thermo Fisher Scientific, DE, USA).

cDNA was produced using the High Capacity cDNA Reverse Transcription Kit (Applied Biosystems, CA, USA) according to the manufacturer's recommendations and cDNA synthesis was performed with the following programme: $25^{\circ} \mathrm{C} 10 \mathrm{~min}(\mathrm{~min}), 37^{\circ} \mathrm{C} 120 \mathrm{~min}$ and $85^{\circ} \mathrm{C} 5 \mathrm{~min}$.

mRNA levels were measured by quantitative realtime polymerase chain reaction (qRT-PCR) using TaqMan $^{\text {TM }}{ }^{\circledR}$ Gene Expression Assays in 7900HT RealTime PCR System (Applied Biosystems, CA, USA). In all samples, Dicer (Hs00229023_m1) and Drosha (Hs00203008_m1) expression levels were normalised to the endogenous housekeeping gene GUSb (Hs99999908_m1) using the $\Delta C T$ calculation. Three technical replicates of all samples were performed and analysed; ddH2O, as non-template control, was analysed for every reaction mix. PCR cycling included the following steps: 1 cycle at $95{ }^{\circ} \mathrm{C}$ for $10 \mathrm{~min}, 45$ times at $95^{\circ} \mathrm{C} 15 \mathrm{~s}$ and $60^{\circ} \mathrm{C}$ for $1 \mathrm{~min}$. Dicer and Drosha relative expression in all tumour samples was subsequently normalised to their median expression values in normal mucosa using the $\Delta \Delta C T$ calculation. ${ }^{17}$

To confirm Dicer protein expression, immunohistochemistry with anti-DICER1 antibody (HPA000694, Sigma) was performed.

\subsection{Statistical analysis}

For all statistical analyses the programme SPSS 17.0 (SPSS, Chicago) was used. The final mRNA levels were converted to ratios of decreased expression $(\leqslant 1)$ or increased expression $(>1)$ relative to levels of Dicer and Drosha mRNA in healthy mucosa. ${ }^{10}$ Student's $t$ test was used to examine the differences in Dicer and Drosha mRNA levels between samples. Student's $t$-test or one-way ANOVA method was applied to analyse the relationships with clinical outcome. Kaplan-Meier method was used to depict survival curves and Cox's Proportional Hazard Model estimated the correlation between mRNA expression value and patients survival in univariate and multivariate analyses. $P$-values $<.05$ were considered statistically significant.

\section{Results}

\subsection{DNA microarray data}

By querying for Dicer (probe 213229_at, Human Genome U133 Plus 2.0 Array) we obtained a total of 29 eligible comparison analyses (cancer versus normal) across several types of cancers whose three analyses were available for $\mathrm{CRC}$, all derived from the series published by Kaiser et al. Fig. S1 shows Dicer mRNA expression across the samples in the Kaiser microarray dataset. As shown in Table S1, a statistically significant difference exists in Dicer expression between healthy mucosa and rectal/rectosigmoid adenocarcinomas (including mucinous histotype). In fact, rectal adenocarcinomas overexpress Dicer with a fold change $>2$ versus normal mucosa. The expression of Dicer in colon adenocarcinomas is also significantly up-regulated compared to normal colon mucosa, but at lesser extent (fold change 1.7-2.0) (Table S1). ${ }^{18}$ By querying for Drosha we obtained only seven eligible comparison analyses (cancer versus normal) across several types of cancers, of which none was available for CRC.

\subsection{Experimental phase: patient population}

We retrospectively selected 116 consecutive patients treated with Bevacizumab-containing regimens for advanced CRC, 50 consecutive patients affected by advanced CRC not treated with Bevacizumab-containing regimens and 20 consecutive patients who underwent 
Table 1

Main clinical characteristics of Bevacizumab treated/untreated patients with advanced CRC.

\begin{tabular}{|c|c|c|}
\hline Patients characteristics & Beva Pts \# $(\%)$ & $\begin{array}{l}\text { Non Beva Pts \# } \\
(\%)\end{array}$ \\
\hline Total number & $116(100)$ & $50(100)$ \\
\hline $\mathrm{M} / \mathrm{F}$ & $62 / 54(53 \% / 47 \%)$ & $39 / 21(78 \% / 22 \%)$ \\
\hline \multicolumn{3}{|l|}{$\operatorname{Age}(y s)$} \\
\hline Median & 64 & 63 \\
\hline Range & $23-81$ & $34-79$ \\
\hline \multicolumn{3}{|c|}{ Performance status (Karnofsky) } \\
\hline Median & 80 & 80 \\
\hline Range & $50-100$ & $40-100$ \\
\hline \multicolumn{3}{|l|}{ Primary tumour site } \\
\hline Colon & $78(67 \%)$ & $32(64 \%)$ \\
\hline Rectum & $38(33 \%)$ & $18(36 \%)$ \\
\hline \multicolumn{3}{|l|}{ No. of metastatic sites } \\
\hline 1 & $49(42 \%)$ & $21(42 \%)$ \\
\hline 2 & $43(37 \%)$ & $18(36 \%)$ \\
\hline$\geqslant 3$ & $24(21 \%)$ & $11(22 \%)$ \\
\hline \multicolumn{3}{|l|}{ Prior adjuvant therapy } \\
\hline None & $29(26 \%)$ & $12(24 \%)$ \\
\hline $\mathrm{FU} / \mathrm{LV}^{\mathrm{a}}$ & $40(34 \%)$ & $23(46 \%)$ \\
\hline FOLFOX regimen & $47(40 \%)$ & $15(30 \%)$ \\
\hline \multicolumn{3}{|l|}{ First line regimen } \\
\hline Bevacizumab + FOLFOX & $69(59 \%)$ & \\
\hline Bevacizumab + FOLFIRI & $47(41 \%)$ & \\
\hline FOLFOX & - & $28(56 \%)$ \\
\hline FOLFIRI & - & $22(44 \%)$ \\
\hline $\begin{array}{l}\text { Cumulative median PFS } \\
\text { (ITT population), mo }\end{array}$ & $\begin{array}{l}11.5 \\
(95 \% \text { CI } 10.88- \\
12.11)\end{array}$ & $\begin{array}{l}8.8 \\
(95 \% \text { CI 5.97-9.9) }\end{array}$ \\
\hline $\begin{array}{l}\text { Cumulative median OS, } \\
\text { mo }\end{array}$ & $\begin{array}{l}28 \\
(95 \% \text { CI } 24.54- \\
31.45)\end{array}$ & $\begin{array}{l}18.9 \\
(95 \% \text { CI 12.19- } \\
29.30)\end{array}$ \\
\hline
\end{tabular}

Abbreviations: CI, confidence interval; F, female; FU, fluorouracil; ITT, intention to treat; LV, leucovorin; M, male; mo, months; OS, overall survival; PFS, progression free survival; ys, years.

a Symbols: according to Mayo Clinic or De Gramont schedules. colorectal surgery for diverticulosis (control group). All 116 patients who received Bevacizumab-based regimens underwent radiological restaging. Moreover we collected peritumoural samples from 53 of the 116 consecutive patients who received Bevacizumab-containing regimens. Table 1 summarises the clinico-pathological features of the 116 and 50 patients treated/untreated with Bevacizumab-containing regimens respectively.

\subsection{Dicer and Drosha $m R N A$ expression in normal mucosa, peritumoural margins and primary tumours in Bevacizumab-treated patients}

The mRNA levels of Drosha in peritumoural margins $(n=53$, median $=19.19$, CI 95\% 16.27-21.11) and primary tumour $(n=116$, median $=24.55$, CI $95 \% 20.40$ 40.59) were significantly increased $(P=.04$ and $<.001$, respectively) compared to the levels in normal mucosa samples resected from patients with diverticulosis (med$\operatorname{ian}=12.45$, CI 95\% 4.32-16.11). Conversely, Dicer expression levels did not show any significant difference between normal mucosa (median $=1,42$ ) and peritumoural $($ median $=1,25 ; P=.09)$ or tumoural samples (median $=1,28 ; P=.080)$.

Dicer protein expression was confirmed by immunohistochemistry (Fig. 1).

\subsection{Dicer and Drosha association with clinical outcome in Bevacizumab-treated patients}

A statistically significant correlation was found between Dicer expression levels and PFS $(P<.0001$, CI $95 \% 10,8-12.1)$ and OS $(P=.009$, CI $95 \% 24.5-$ $31.4)$ in Bevacizumab-treated patients with advanced CRC (Table 2). These results suggested a strong association between low Dicer levels and favourable outcome, in terms of PFS and OS. On the other hand, Drosha
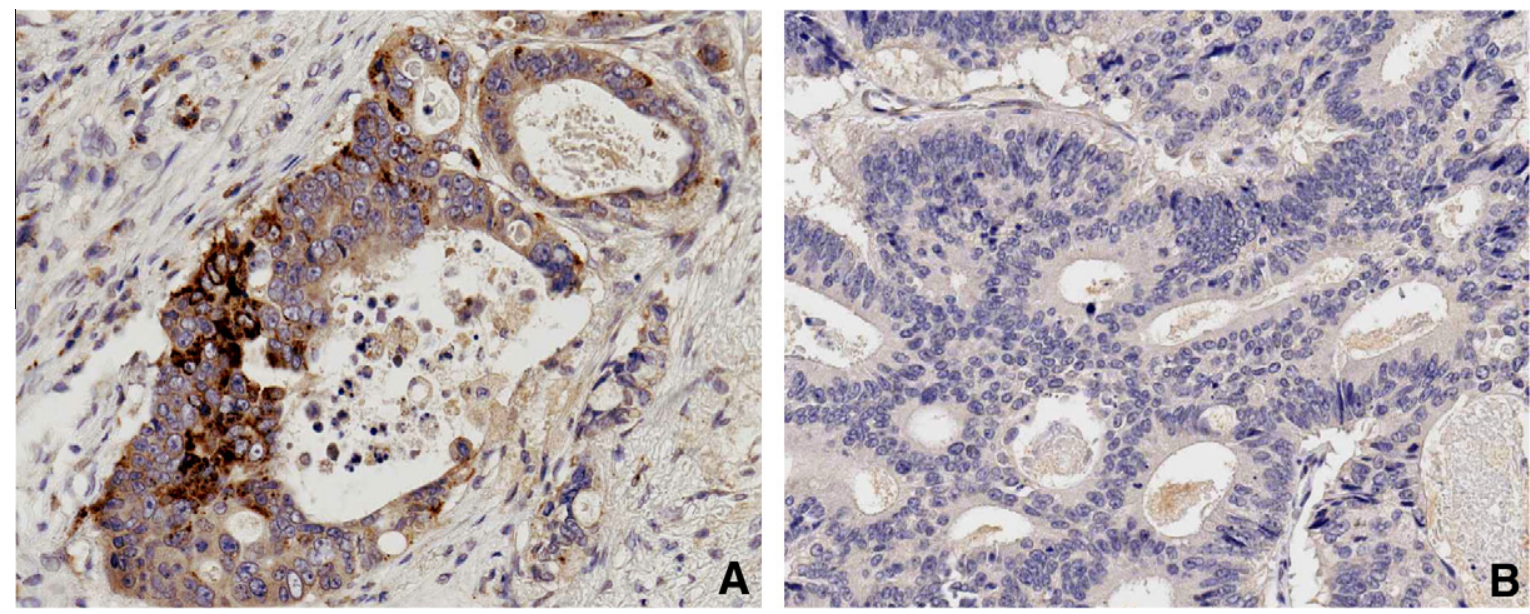

Fig. 1. Dicer expression in human colon cancer. Colon cancer with high level of mRNA Dicer expression shows significant staining in tumour cell cytoplasm for anti-Dicer1 antibody (A). Differently, colon cancer with low level of mRNA Dicer expression was negative for anti-Dicer1 immunoreaction (B). Original magnification $200 \times$. 
Table 2

Dicer and Drosha expression levels and clinical outcome.

\begin{tabular}{lll}
\hline Dicer mRNA level & Median PFS $(95 \% \mathrm{CI}) \mathrm{mo}$ & $P$ value \\
\hline Low & $13.2(11.77-14.62)$ & $<.0001$ \\
High & $9.7(9.03-10.46)$ & \\
& \multicolumn{1}{l}{ Median OS $(95 \% \mathrm{CI}) \mathrm{mo}$} & \\
Low & $31.0(25.52-36.47)$ & \\
High & $24.0(19.89-28.10)$ & $=.254$ \\
Drosha mRNA level & Median PFS $(95 \% \mathrm{CI}) \mathrm{mo}$ & \\
\cline { 2 - 2 } Low & $12.8(11,81-13.78)$ & $=.262$ \\
High & $10.0(8.93-11.06)$ & \\
\cline { 2 - 2 } & $28.0(24.71-31.28)$ & \\
Low & $25.0(21.63-28.36)$ & \\
High &
\end{tabular}

Abbreviations: CI, confidence interval; mo, months; PFS, progressionfree survival; OS, overall survival.

expression levels did not show any statistically significant correlation with clinical outcome (PFS: $P=.254$, CI $95 \% 10.8-12.1$; OS: $P=.262$, CI $95 \% 24.5-31.4$ ) (Fig. 2; Table 2). Additionally, the response to Bevacizumab-based treatment according to RECIST criteria was evaluated on the basis of Dicer/Drosha levels. Patients with low Dicer expression levels showed a better response to Bevacizumab-based therapy $(1.7 \%$ complete responses (CR) and 53.4\% partial responses (PR); $P=.0067)$ compared to patients with high Dicer mRNA levels ( $0 \% \mathrm{CR}$ and $32.7 \% \mathrm{PR})$, as shown in Table 3 . The same results were not observed for Drosha.

In the multivariate analysis that included PS, number of organs involved, carcinoembryonic antigen and low dicer, the relationship between low Dicer and survival remained significant (PFS: $P=.006, \mathrm{RR}=0.48,95 \%$ CI $0.33-0.81$; OS: $P=.008, \mathrm{RR}=0.55,95 \%$ CI 0.40 0.86 for) as shown in Table 4. Other prognostic variables tested such as tumour grading, liver involvement, albumin, alkaline phosphatase, gamma-GT and LDH have not shown statistical significance.

\subsection{Dicer and Drosha association with clinical outcome in patients not treated with Bevacizumab-based therapy}

In order to assess the potential role of Dicer and Drosha in predicting efficacy of Bevacizumab-based treatments, we analysed Dicer and Drosha expression levels in a control group of patients affected by advanced $\mathrm{CRC}$ not treated with Bevacizumab-containing regimens. In this group of patients, their expression levels did not show any statistically significant correlation with survival (Table 5) suggesting a potential predictive role of Dicer in response to the anti-angiogenetic based treatment.

\section{Discussion}

Several preclinical evidences elucidated the role of the two key enzymes for miRNA biogenesis Dicer and
Drosha in angiogenesis and endothelial function. In a model of human endothelial cells (ECs) the Dicer or Drosha silencing leads to a slight decrease in angiogenesis, evaluated by endothelial tube formation in matrigel. ${ }^{13}$ In a mouse Dicer knock-down model, it has been demonstrated that endothelial miRNAs are required for postnatal angiogenesis in response to angiogenic stimuli. Indeed this model showed a decreased postnatal angiogenesis after Dicer knock-down in response to different stimuli such as exogenous VEGF, tumours, ischaemia and wound healing. 13,19-21 Interestingly, Kuehbacher et al. found that migration of ECs was significantly decreased in Dicer siRNA-transfected cells, whereas Drosha siRNA had no effect. ${ }^{13}$ Silencing of Dicer but not of Drosha seems to reduce angiogenesis in vivo.

Starting from these preclinical data is reasonable to think that differential expressions of Dicer and to a lesser extent Drosha in cancer patients could influence the outcome and the efficacy of treatments based on angiogenesis inhibitors. To investigate these hypotheses we evaluated whether Dicer and Drosha showed different mRNA expression levels in $116 \mathrm{CRC}$ samples and 20 normal mucosa tissues from patients with diverticulosis. Moreover, we analysed peritumoural sections from 53 of the 116 tumour samples in order to investigate if they had a similar expression profile compared to normal mucosa samples from patients resected for diverticular disease. We found a statistically significant increase in the median mRNA levels of Drosha in tumour sections compared to peritumoural samples and healthy mucosa. It is presumable that the peritumoural tissue, which is assumed to be not impaired during cancer formation and apparently normal at morphological level, could show abnormal gene regulation in comparison to normal mucosa from non-cancer patients supporting its involvement both in early cancer formation and in the progression. However, these data need further investigation. Conversely, we did not found any significant difference in Dicer expression levels among our three groups of samples.

Stratmann et al. found that Dicer expression was increased in primary tumours in comparison to that in normal mucosa from rectal cancer patients but this was not evident in colon cancer patients; moreover its expression was higher in rectal cancer than in colon cancer. $^{12}$

Analysing the Bevacizumab treated patient group we interestingly observed that low Dicer expression was associated with a favourable outcome in terms of PFS and OS, independently of other clinical parameters. Indeed patients with low Dicer expression levels presented a better response to Bevacizumab-based therapies compared to patients with high Dicer levels. These data are in accordance with previous studies that showed a direct involvement of Dicer in the development and clinical outcome of malignancies such as ovarian, prostate 
A

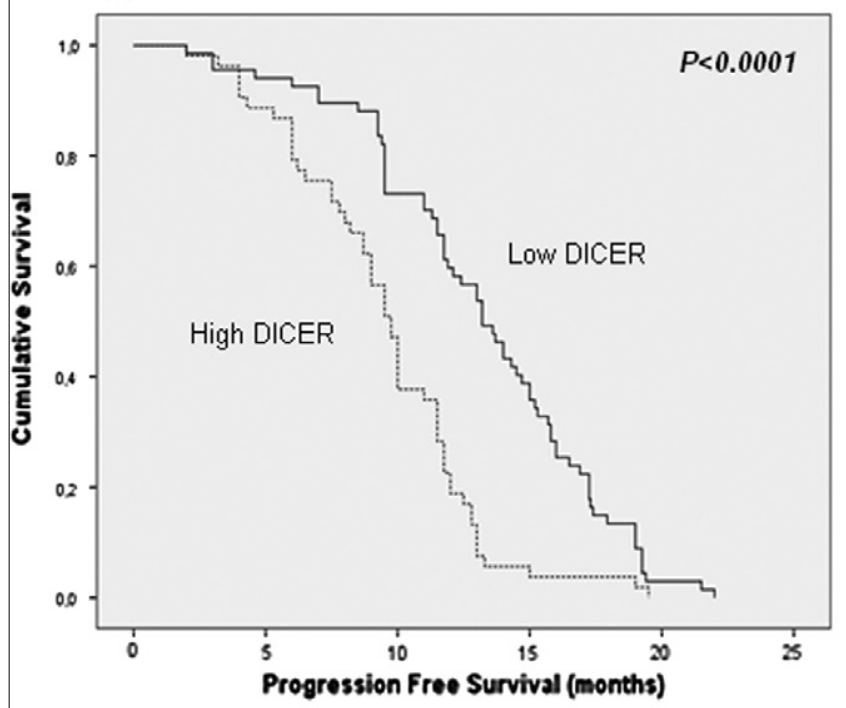

C

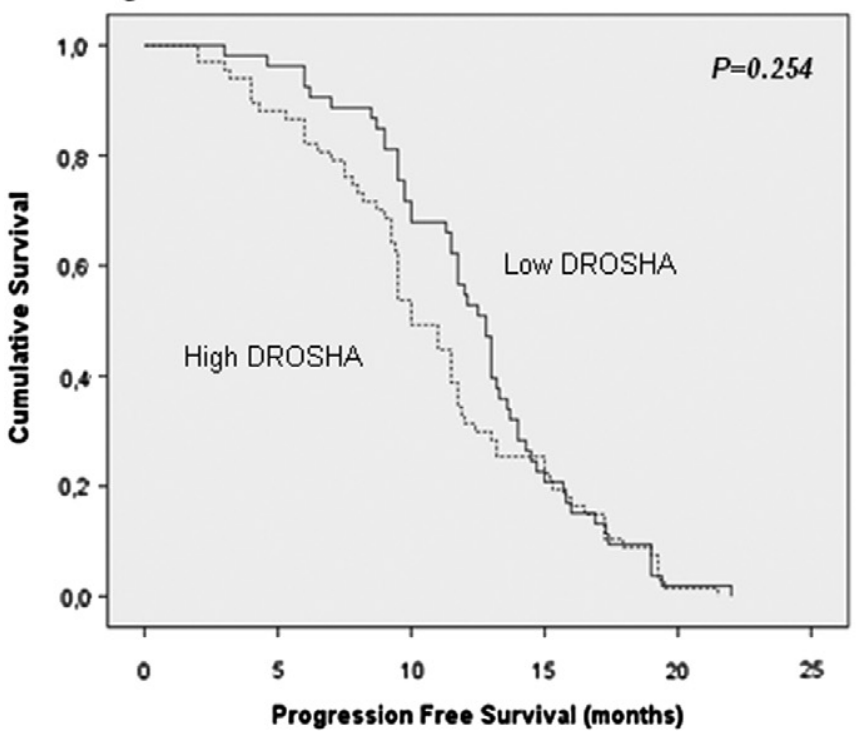

B

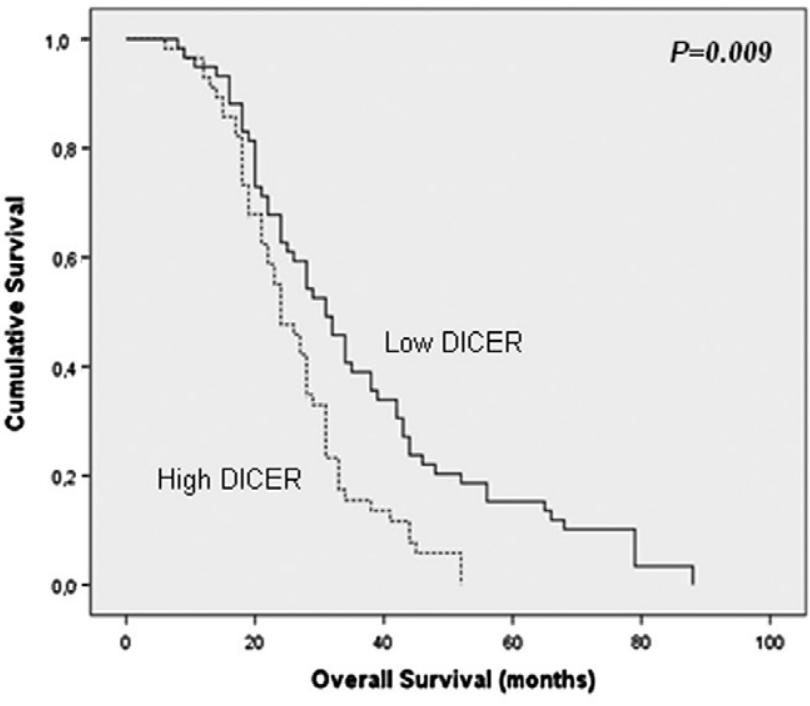

D

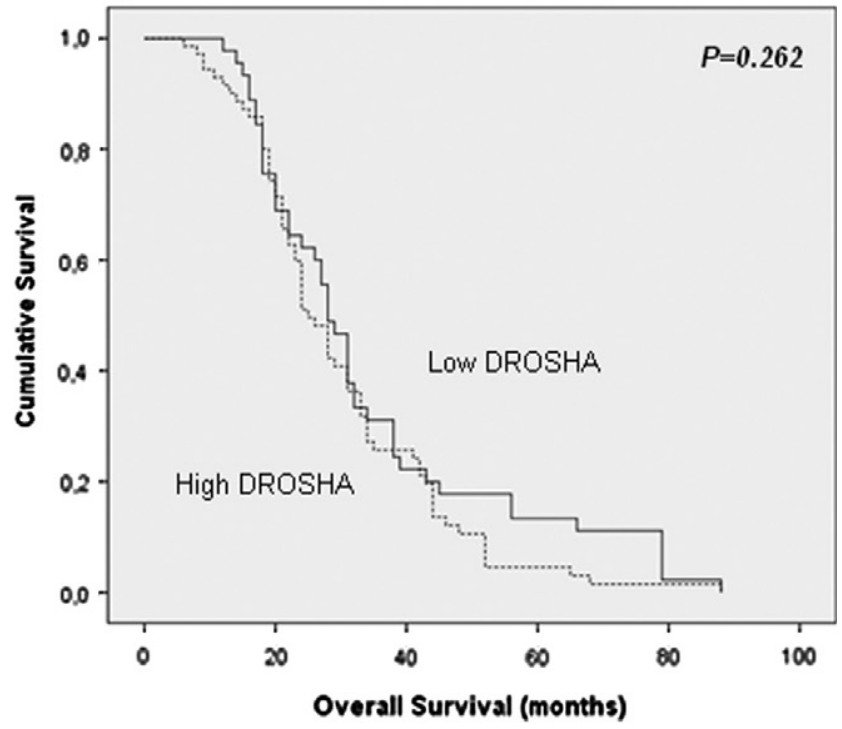

Fig. 2. Kaplan-Meier curves of PFS (panel A) and OS (panel B) show that low Dicer expression levels correlate with longer PFS $(P<.0001)$ and OS $(P=.009)$ in patients with mCRC treated with Bevacizumab containing regimens; Kaplan-Meier curves of PFS (panel C) and OS (panel D) show that low/high Drosha expression is not significantly associated to difference in terms of OS and PFS in patients with mCRC treated with Bevacizumab containing regimens.

Table 3

DICER and DROSHA expression and clinical response. ${ }^{a}$

\begin{tabular}{lllll}
\hline mRNA level & \# CR (\%) & \# PR (\%) & \# SD (\%) & \# PD (\%) \\
\hline Low Dicer & $1(1.7 \%)$ & $31(53.4 \%)$ & $15(25.8 \%)$ & $11(18.9 \%)$ \\
High Dicer & $0(0 \%)$ & $19(32.7 \%)$ & $20(34.4 \%)$ & $19(32.7 \%)$ \\
Low Drosha & $1(1.7 \%)$ & $27(46.5 \%)$ & $17(29.3 \%)$ & $13(22.4 \%)$ \\
High Drosha & $0(0 \%)$ & $23(39.6 \%)$ & $18(31 \%)$ & $17(29.3 \%)$ \\
\hline
\end{tabular}

Abbreviations: CR, complete response; PD, progressive disease PR, partial response; SD, stable disease.

${ }^{a}$ According to RECIST (1.0) criteria.

and breast cancer. ${ }^{10,22,23}$ On the other hand, Drosha expression did not significantly correlate with prognosis.
However, analysing the Dicer expression levels in the 50 patients untreated with Bevacizumab-based regimens 
Table 4

Multivariate analysis for PFS and OS.

\begin{tabular}{|c|c|c|c|c|c|c|}
\hline \multirow[t]{2}{*}{ Factors } & \multicolumn{3}{|c|}{ Univariate } & \multicolumn{3}{|c|}{ Multivariate } \\
\hline & $\mathrm{RR}$ & $95 \% \mathrm{CI}$ & $\mathrm{P}$ & $\mathrm{RR}$ & $95 \% \mathrm{CI}$ & $\mathrm{P}$ \\
\hline \multicolumn{7}{|l|}{$P F S$} \\
\hline PS (70-80 versus $81-100)$ & 1.31 & $1.06-1.76$ & .034 & 1.15 & $0.67-1.35$ & .322 \\
\hline $\mathrm{N}$ organs involved ( 1 versus more) & 0.82 & $0.63-0.96$ & .021 & 0.95 & $0.61-0.98$ & .047 \\
\hline Low Dicer & 0.48 & $0.27-0.69$ & $<.0001$ & 0.67 & $0.33-0.81$ & .006 \\
\hline \multicolumn{7}{|l|}{$O S$} \\
\hline PS (70-80 versus $81-100)$ & 1.91 & $1.22-2.51$ & .004 & 1.45 & $1.16-2.23$ & .011 \\
\hline $\mathrm{N}$ organs involved ( 1 versus more) & 0.68 & $0.51-0.91$ & .008 & 0.79 & $0.60-0.95$ & .034 \\
\hline Low Dicer & 0.55 & $0.32-0.87$ & 0.003 & 0.71 & $0.40-0.86$ & .008 \\
\hline $\mathrm{CEA}^{\mathrm{a}}$ & 1.31 & $1.05-1.97$ & .035 & 1.09 & $0.88-1.41$ & .318 \\
\hline
\end{tabular}

Abbreviations: CEA, carcinoembryonic antigen; CI, confidence interval; N, number; PFS, progression-free survival; PS, performance status; RR, relative risk; OS, overall survival.

${ }^{a}$ Symbols: continuous variable for every 50-point CEA increase.

Table 5

Dicer and Drosha expression levels and patient outcome in the control group (FOLFOX IV).

\begin{tabular}{|c|c|c|}
\hline Dicer mRNA level & Median PFS $(95 \% \mathrm{CI}) \mathrm{mo}$ & $P$ value \\
\hline Low & $9.8(5.06-14.1)$ & .639 \\
\hline \multirow[t]{2}{*}{ High } & $8.7(4.87-10.84)$ & \\
\hline & Median OS $(95 \% \mathrm{CI}) \mathrm{mo}$ & \\
\hline Low & $24.0(18.20-31.88)$ & .416 \\
\hline High & $21.4(16.99-27.01)$ & \\
\hline Drosha mRNA level & Median PFS $(95 \% \mathrm{CI}) \mathrm{mo}$ & $P$ value \\
\hline Low & $9.4(5.98-13.02)$ & .860 \\
\hline \multirow[t]{2}{*}{ High } & $8.6(5.41-11.97)$ & \\
\hline & Median OS $(95 \% \mathrm{CI}) \mathrm{mo}$ & \\
\hline Low & $23.9(17.22-27.17)$ & .761 \\
\hline High & $22.8(16.84-26.10)$ & \\
\hline
\end{tabular}

Abbreviations: CI, confidence interval; mo, months; PFS, progressionfree survival; OS, overall survival.

we did not find a statistically significant association with patient survival.

These data do not seem to support a possible Dicer prognostic role in $\mathrm{mCRC}$, as previously published in literature, suggesting, hence, a potential role of Dicer as a biological parameter that could predict the efficacy of an anti-angiogenetic therapy. ${ }^{11}$

As mentioned above, data from literature show that Dicer could be directly involved in the regulation of VEGF-dependent angiogenetic processes. Preclinical results indicate that transient reduction of the miRNA-regulating enzyme Dicer impairs angiogenesis in vitro and in vivo, whereas Drosha siRNA induced a minor antiangiogenic effect in vitro and is not effective in vivo. ${ }^{13}$ Specifically, Dicer down-regulation demonstrated to induce an increase in expression level of several key genes involved in regulation of endothelial biology and angiogenesis, such as TEK/Tie2, KDR/ VEGFR2, Tie-1, eNOS and IL-8. ${ }^{21}$ Moreover, Dicer silencing leads to strong up-regulation of the potent angiogenesis inhibitor thrombospondin-1 (Tsp-1), identified also by in silico analysis. ${ }^{13}$ Tsp-1 is a predicted tar- get of the Let-7 family and the miR-17-92 cluster and Dicer and Drosha siRNA reduced lef-7f and miRNA$27 \mathrm{~b}$ expression. ${ }^{13}$ Interestingly, inhibitors of Let-7f, miRNA-17-92 and miRNA-27b clusters reduce EC sprouting and matrigel tube formation in vitro, indicating that these miRNAs promote angiogenesis by targeting antiangiogenic genes. ${ }^{13,21}$ Notably, by looking at the most co-expressed genes with Dicer in Kaiser microarray dataset, our analysis identified among the top 15 ranked genes ATRX and NKTR (correlation 0.59 for both), both targeted by miRNA-27b. ${ }^{19}$

On the basis of these evidences, our clinical study provides an initial support to the hypothesis of an increased efficacy of Bevacizumab-based treatments in patients with low levels of Dicer and consequent impairment of angiogenesis pathways. This correlation is not observed for Drosha, confirming both clinical and preclinical results.

Further studies are also warranted to deeper investigate the relationship between potential Dicer-dependent targets (e.g. let-7 family and mir-27b) and angiogenesis modulation in CRC patients. Moreover in order to confirm these very preliminary results, we consider mandatory their validation into prospective and larger translational studies. If confirmed, these data would further support the use of Dicer expression level as predictive marker of response to Bevacizumab-based therapy in advanced $\mathrm{CRC}$, helping the clinicians to choose the optimal personalised therapy for advanced CRC patients.

\section{Conflict of interest statement}

The authors declare no conflict of interest.

\section{References}

1. Ferlay J, Autier P, Boniol M, Heanue M, Colombet M, Boyle P. Estimates of the cancer incidence and mortality in Europe in 2006. Ann Oncol 2007;18:581-92. 
2. Fearon ER. Molecular genetics of colorectal cancer. Annu Rev Pathol 2011;6:479-507.

3. Carthew RW, Sontheimer EJ. Origins and mechanisms of miRNAs and siRNAs. Cell 2009;136:642-55.

4. Lee Y, Ahn C, Han J, et al. The nuclear RNase III Drosha initiates microRNA processing. Nature 2003;425:415-9.

5. Bernstein E, Caudy AA, Hammond SM, Hannon GJ. Role for a bidentate ribonuclease in the initiation step of RNA interference. Nature 2001;409:363-6.

6. Grishok A, Pasquinelli AE, Conte D, et al. Genes and mechanisms related to RNA interference regulate expression of the small temporal RNAs that control C. elegans developmental timing. Cell 2001;106:23-34.

7. Lee Y, Jeon K, Lee JT, Kim S, Kim VN. MicroRNA maturation: stepwise processing and subcellular localization. EMBO J 2002;21:4663-70.

8. Karube Y, Tanaka H, Osada H, et al. Reduced expression of Dicer associated with poor prognosis in lung cancer patients. Cancer $S c i$ 2005;96:111-5.

9. Grelier G, Voirin N, Ay AS, et al. Prognostic value of Dicer expression in human breast cancers and association with the mesenchymal phenotype. Br J Cancer 2009;101:673-83.

10. Merritt WM, Lin YG, Han LY, et al. Dicer, Drosha, and outcomes in patients with ovarian cancer. $N$ Engl $J$ Med 2008;359:2641-50.

11. Faber C, Horst D, Hlubek F, Kirchner T. Overexpression of Dicer predicts poor survival in colorectal cancer. Eur $J$ Cancer 2011;47:1414-9.

12. Stratmann J, Wang CJ, Gnosa S, et al. Dicer and miRNA in relation to clinicopathological variables in colorectal cancer patients. BMC Cancer 2011;11:345.

13. Kuehbacher A, Urbich C, Zeiher AM, Dimmeler S. Role of Dicer and Drosha for endothelial microRNA expression and angiogenesis. Circ Res 2007;101:59-68.
14. Yang WJ, Yang DD, Na S, Sandusky GE, Zhang Q, Zhao G. Dicer is required for embryonic angiogenesis during mouse development. J Biol Chem 2005;280:9330-5.

15. Jain RK. Normalizing tumor vasculature with anti-angiogenic therapy: a new paradigm for combination therapy. Nat Med 2001;7:987-9.

16. Presta LG, Chen H, O'Connor SJ, et al. Humanization of an antivascular endothelial growth factor monoclonal antibody for the therapy of solid tumors and other disorders. Cancer Res 1997;57:4593-9.

17. Livak KJ, Schmittgen TD. Analysis of relative gene expression data using real-time quantitative PCR and the 2(-Delta Delta C(T)). Method Methods 2001;25:402-8.

18. Kaiser S, Park YK, Franklin JL, et al. Transcriptional recapitulation and subversion of embryonic colon development by mouse colon tumor models and human colon cancer. Genome Biol 2007;8:R131.

19. Shilo S, Roy S, Khanna S, Sen CK. Evidence for the involvement of miRNA in redox regulated angiogenic response of human microvascular endothelial cells. Arterioscler Thromb Vasc Biol 2008;28:471-7.

20. Suarez Y, Fernandez-Hernando C, Pober JS, Sessa WC. Dicer dependent microRNAs regulate gene expression and functions in human endothelial cells. Circ Res 2007;100:1164-73.

21. Suarez Y, Fernandez-Hernando C, Yu J, et al. Dicer-dependent endothelial microRNAs are necessary for postnatal angiogenesis. Proc Natl Acad Sci U S A 2008;105:14082-7.

22. Chiosea S, Jelezcova E, Chandran U, et al. Up-regulation of dicer, a component of the microRNA machinery, in prostate adenocarcinoma. Am J Pathol 2006;169:1812-20.

23. Dedes KJ, Natrajan R, Lambros MB, et al. Down-regulation of the miRNA master regulators Drosha and Dicer is associated with specific subgroups of breast cancer. Eur J Cancer 2011;47:138-50. 